\title{
Development of VTOL Drone for Stable Transit Flight
}

\author{
Seunghee Yu, Yongjin Kwon* \\ Department of Industrial Engineering, College of Engineering, Ajou University, Suwon, South Korea \\ Email: ${ }^{\star} y k 73 @ a j o u . a c . k r$
}

How to cite this paper: $\mathrm{Yu}, \mathrm{S}$. and Kwon, Y.J. (2017) Development of VTOL Drone for Stable Transit Flight. Journal of Computer and Communications, 5, 36-43. https://doi.org/10.4236/jcc.2017.57004

Received: April 12, 2017

Accepted: May 14, 2017

Published: May 17, 2017

Copyright $\odot 2017$ by authors and Scientific Research Publishing Inc. This work is licensed under the Creative Commons Attribution International License (CC BY 4.0).

http://creativecommons.org/licenses/by/4.0/

\begin{abstract}
Fixed wing unmanned aerial vehicles typically have longer running (flight) time than multicopers, but runways are needed. Because it is difficult to expect a wide runway in the city area, the great need is there to develop a vertical takeoff and landing (VTOL) type fixed-wing UAV that does not require any runway. In addition, our goal was to develop a VTOL plane that contains a stable transit flight characteristic. To achieve this goal, we have designed and developed a test plane and the flight parameters have been extensively tested. For the airframe design, the carbon-bar method was selected for vertical takeoff and landing, and the 11-way method was adopted through dynamics analysis. We assembled the airframe and made a prototype using the Pixhawk flight computer. The developed VTOL plane shows a very smooth transition flight. It is expected that the VOTL UAV will be widely used in the city area in the future for various purposes, such as cargo delivery and emergency medical supply delivery.
\end{abstract}

\section{Keywords}

UAV, Multi-Coper, Fixed Wing, UAV, QUAD PLANE, Pixhawk, Transit Flight

\section{Introduction}

In many industrial fields, the two commonly used UAV types include multi-copter types and fixed-wing types. Multi-copter aircrafts are easy to operate, and vertical takeoff and landing is possible. It is easy to carry out various missions in the relatively congested areas such as city centers that are surrounded by many buildings. However, there are considerable limitations in performing missions such as wide-area surveillance and searcher missions, which require longdistance flying and long-flight times. On the other hand, the fixed-wing UAV has a longer operating range and longer running time than the multi-copter 
UAV. However, it is difficult to take off and land without a separate runway [1]. Therefore, they are almost impossible to operate in the city areas. At present, many companies related to UAVs are developing VTOL types UAVs with the advantages of fixed wings, but most of them are not yet fully commercialized. Internationally, if the VTOL UAVs are successfully commercialized, it will contribute greatly to the creation of a new market.

The VTOL design, however, carried an inherent weakness, in terms of added weight due to extra propulsion systems. The conventional fixed wing aircraft models only need one or two motors to propel the fuselage [2] [3]. The VTOL needs the vertical lift capability, which typically needs at least one or two extra motors, and also needs the horizontal propellers. Some VTOL design incorporates the tilting mechanism. In other words, the vertical lift mechanism is used for both vertical flight as well as horizontal flight, once the aircraft is airborne. This tilting mechanism, however, increases the complexity of the design and added weight. Additionally, during the transition flight (that is, from hovering to horizontal flight), there is a risk in terms of the aircraft that may lose the balance and fall from the sky [4] [5] [6] [7]. Due to these reasons, the VTOL design always has to find the optimal way of constructing light weight and efficient propulsion design. We have conducted a preliminary study and analyzed the various VTOL designs. There is an obvious trend for the VTOL design. Two most common VTOL designs include the fixed wing type design: 1) with tilting mechanism, and 2) without tilting mechanism [8] [9] [10] [11]. As previously mentioned, both approaches have advantages and disadvantages. The tilting mechanism is more efficient, because the motors used for vertical lift are later used for horizontal propulsion, hence reducing the number of onboard motors. For the non-tilting VTOL, the stable and safe transition flight is the merit, with the reduced level of system complexity. With the current level of literature review, it was found that there is not much literature available that addresses the VTOL design, which provides stable transition flight characteristics. Therefore, the purpose of this study is to develop a VTOL UAV, which has a stable transit flight characteristic. In our previous attempts, the transition flight was very difficult to attain. In order for the VTOL UAVs to become commercially successful, they should be flying safely between the vertical hover and the straight flight.

\section{Technology Development Contents}

In this study, we designed and fabricated a miniature model before developing a full-scale VTOL UAV, and tested flight stability and various parameters.

\subsection{Carbon Bar Fixing Method}

VTOL fixed-wing UAVs should be freely convertible to quad-copter mode and fixed-wing mode as needed. Therefore, four vertical propellants and one horizontal propellant are mounted on the fixed-wing aircraft. First, a car-bon bar system for fixing four propellants for vertical flight is defined. Each carbon bar is equipped with two propellants and the bar is fixed in two ways [12]. 
1) X-way Fixed bar

2) 11-way Fixed bar

\subsection{Fuselage Design}

Based on the two-axis fixation method mentioned above, design work was carried out using Solidworks CAD software.

\subsubsection{X-Way Fixed Bar}

The X-bar method is applied to the propeller shaft fixing method of most vertical take-off drones. Additional supports were designed to stabilize the bar intersecting the $\mathrm{X}$ bars. Figure 1 illustrates the design drawing as well as the actual construction of X-shaped propulsion system.

\subsubsection{1-Way Fixed Bar}

The 11-character scheme is called the Quadplane. We did not design the support bar separately because it could be attached directly to the carbon bar mounted on the wing part. Figure 2 shows the actual construction of the design and the final assembly.

\subsubsection{Comparison of Bar Fixing Methods}

There are three obstacles to be considered in the selection of the locking method of the bar.

1) Elements resistant to translational motion

2) Increased weight due to additional equipment

3) Elements that give fatigue to gas parts

Compared with the $\mathrm{X}$ method, the 11-way method is less affected by the maneuvering caused by the rotational motion of the propeller because the vertical sectional area is reduced in the traveling direction. In this way, the drag is rela-

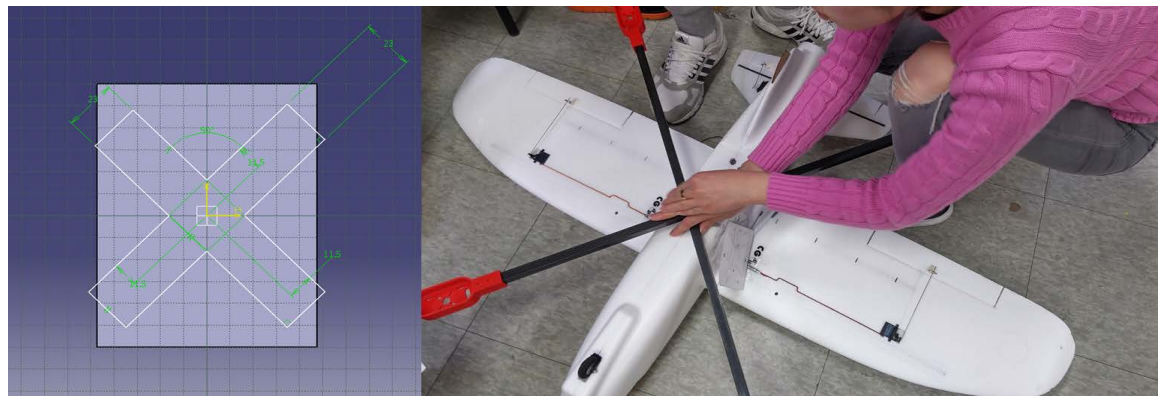

Figure 1. X-shaped fuselage design and bar support fixture.

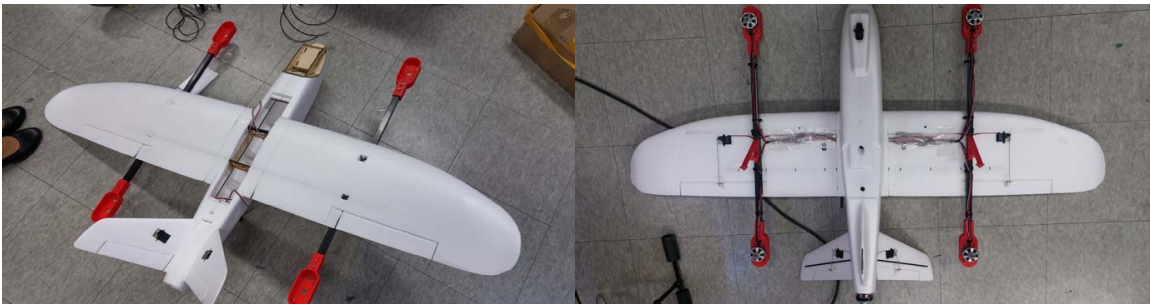

Figure 2. 11-way fuselage design. 
tively small and the bars are parallel to each other. In addition, there is no need to install additional support, and there is no weight increase due to additional equipment. However, because it is mounted directly on the wing, it is possible to cause fatigue cracks as the flight time elapses [13].

\subsubsection{Dynamic Analysis}

The force acting on the aircraft consists of gravitational acceleration, positive force $\left(F_{\text {aero }}^{b}\right)$, and thrust $\left(F_{\text {th }}^{b}\right)[1][12][13]$.

$$
\begin{gathered}
F^{b}=F_{g}^{b}+F_{\text {aero }}^{b}+F_{\text {th }}^{b} \\
F^{b}=\left[\begin{array}{l}
F_{x} \\
F_{y} \\
F_{z}
\end{array}\right] \quad F_{\text {th }}^{b}=\left[\begin{array}{l}
T \\
0 \\
0
\end{array}\right] \quad F_{\text {aero }}^{b}=\mathrm{Q} 5\left[\begin{array}{l}
C_{x} \\
C_{y} \\
C_{z}
\end{array}\right]
\end{gathered}
$$

The thrust corresponding to the force of the motor has a direct influence only on the force $F_{x}$ that the air travels forward. Here, it is necessary to take a good look at the lift. The longitudinal aerodynamic coefficients $C_{x}$ and $C_{z}$ affecting the longitudinal motion of the aircraft are decomposed as lift and aerodynamic forces $C_{L}$ and $C_{D}$ as follows.

$$
\left[\begin{array}{l}
C_{X} \\
C_{Z}
\end{array}\right]=\left[\begin{array}{cc}
\sin \theta & -\cos \theta \\
-\cos \theta & -\sin \theta
\end{array}\right]\left[\begin{array}{l}
C_{L} \\
C_{D}
\end{array}\right]
$$

Since the angle of attack of the aircraft is $0^{\circ}<\theta<45^{\circ}$, the value of $C_{L}$ must be large and the value of $C_{D}$ must be very small in order to increase the $C_{x}$ coefficient for longitudinal forward motion.

$$
F_{D}=\frac{1}{2} \rho V^{2} C_{D} A
$$

Considering only the drag force component, $F_{D}$ is the drag $(\mathrm{N}), \rho$ is the density of the gas $\left(\mathrm{kg} / \mathrm{m}^{3}\right), V$ is the relative velocity between gas and solid $(\mathrm{m} / \mathrm{s})$, drag coefficient and $A$ is perpendicular to the relative velocity, and the area of the cross-sectional area $\left(\mathrm{m}^{2}\right)$. Here, the drag increases as the vertical cross-sectional area of the bar is added to the direction of the designed airframe. The $\mathrm{X}$-bar fixing method having a very large vertical cross-sectional area is problematic due to the large drag [14] [15].

\section{Prototype Production}

The position of the carbon bar in the wing and the line that does not invade the V-TAIL (tail wing) area was determined, while being positioned close to the square to allow stable flight. The Pixhawk flight controller (FC) is installed inside the plane and each motor is connected as well as the electronic speed controllers (ESC). In addition, GPS, receiver module, and switches are installed. They are installed inside the aircraft, so they were placed as close as possible to the center of the airframe, which had a relatively low impact due to fear of affecting flight capability such as air resistance during the flight [14]. Figure 3 shows the completed VTOL design with the 11-shaped motor fixture. 


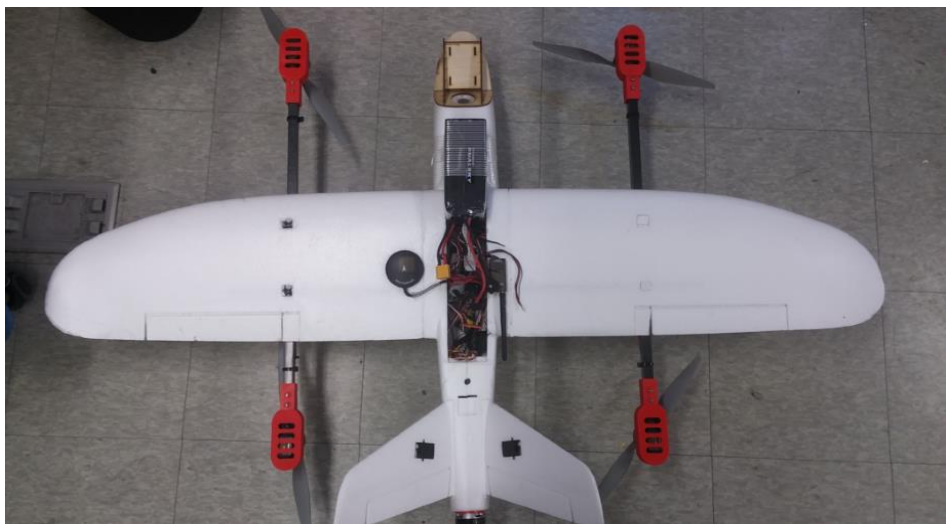

Figure 3. Completed VTOL Drone.

\section{Flight Parameter Setting}

We used the Pixhawk program to set the quad plane. The setting process is as follows.

1) We used a mission planner to set the Firmware of Pixhawk

2) The following is the "Accelerometer calibration" for flying. This accelerometer calibration is an essential process to use the Extended Kalman Filter (EKF). It is mainly used in plane to measure speed, accelerometer, GPS, COMPASS, gyroscope and so on. After that, you can set the available flight mode on the quad plane and set the "Compass calibration" to complete the essential program settings

3) Figure 4 graphically illustrates the setting screen for the VTOL flight parameters. Through the various setting options, we could set the PID (proportional, integrative derivative) gain values that provide the most optimal combination of response characteristics. The PID gain setting was very crucial for the stable flight.

\section{Test Flight and Inspection}

After verifying the operation of the manufactured VTOL gas with the receiver, the test flight was performed in the backyard of the school. Figure 5 shows the vertical takeoff and horizontal flight. The test was very successful and the aircraft showed a very stable flight characteristic.

Large vibrations degrade the performance of precise location-based modes and cause flight instability when vertically landing in quad-copter mode. It is judged that there is no problem when the average vibration shows average 15 $\mathrm{m} / \mathrm{s} / \mathrm{s}$ and maximum $30 \mathrm{~m} / \mathrm{s} / \mathrm{s}$. Unlike the other axes, the $\mathrm{Z}$ axis has a maximum deviation of 36.07 and the minimum deviation of 1.29 , which requires a separate damping action. The CLIP value is a cumulative value that increases when one of the accelerometers reaches the maximum value (16 G). During the flight, the CLIP values need to stay around zero, yet can be climbing up to 100. In our test, the CLIP values remained largely around zero values, hence representing a stabile flight. When checking the clip graph, it was found that all of values were overlapped with each other, which was quite desirable [15] [16]. Figure 6 shows 


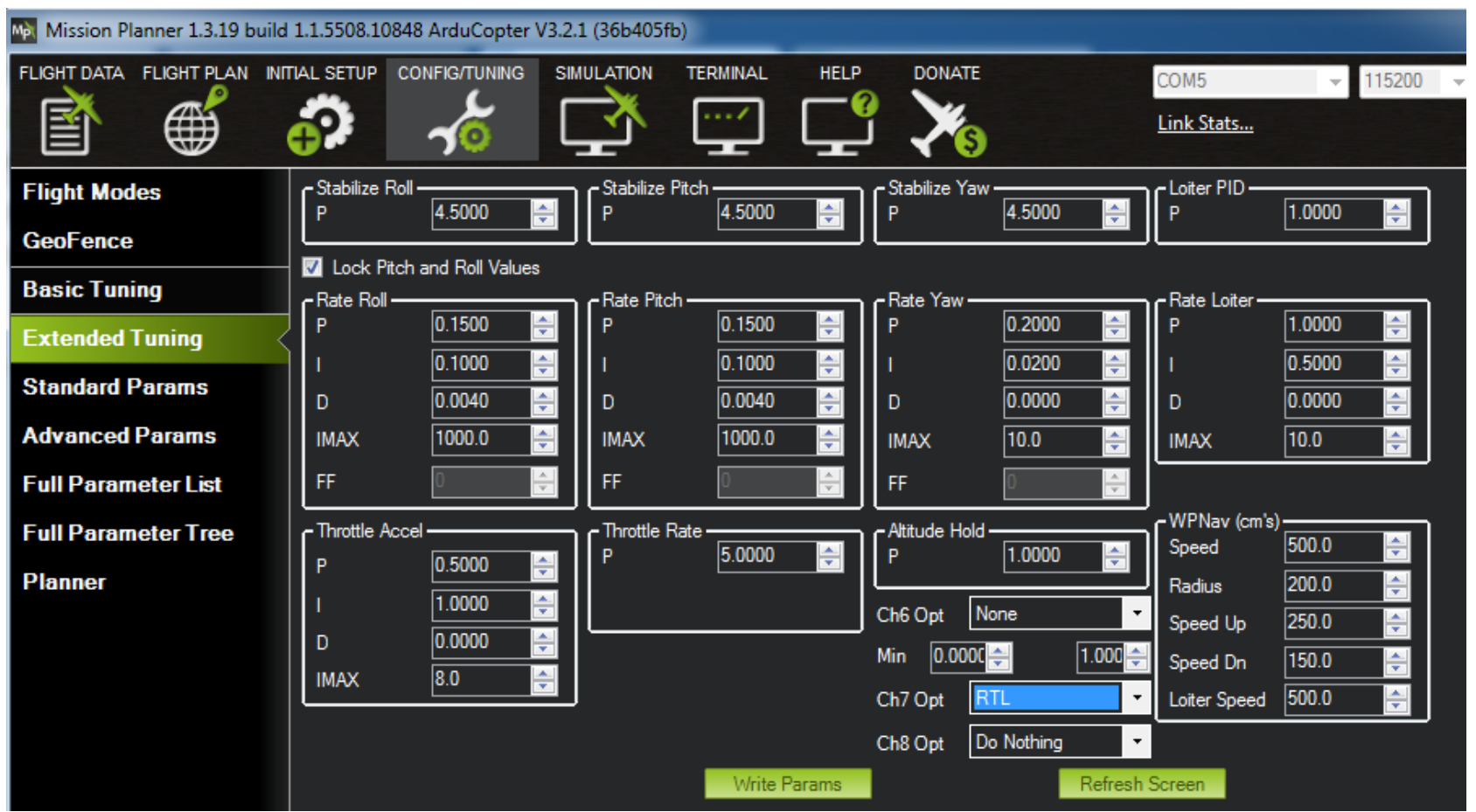

Figure 4. Mission planner setting screen.

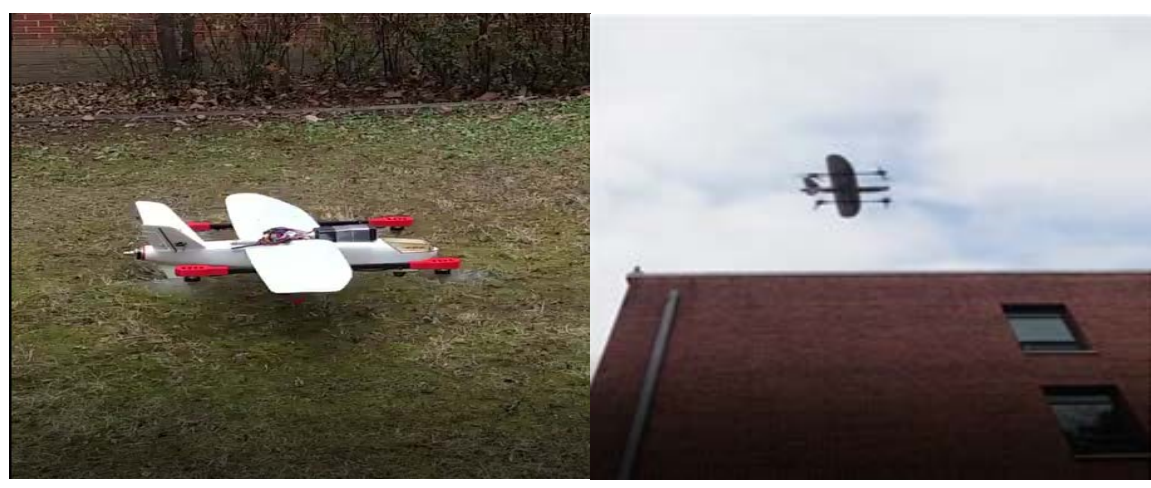

Figure 5. Test flight.

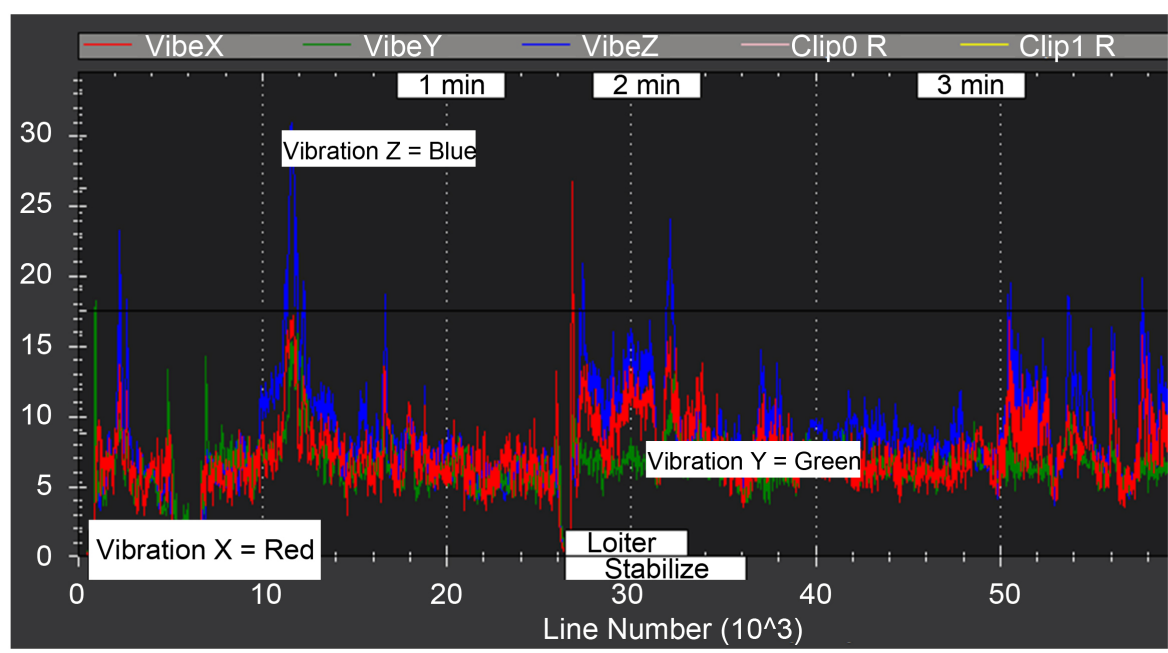

Figure 6. VIBE graph. 
the actual vibration values along $\mathrm{X}, \mathrm{Y}$, and $\mathrm{Z}$ axes of the aircraft. As a result, the transition flight was very stable and smooth. Consequently, the aircraft was deemed suitable for utilizing the developed parameters. It is expected that the $\mathrm{UAV}$ can be used in the congested areas such as city centers in the near future.

\section{Conclusions}

In this study, we have designed and developed a VTOL fixed-wing unmanned aerial vehicle capable of overcoming shortcomings of multi-copter flight and shortcomings of geographical conditions in city areas due to the rapid increase of the unmanned aerial vehicle market. Before designing the product, the quality and the characteristics of the technology and the technology required through the QFD analysis were derived and the priority was taken into account. CAD and Solidworks design and dynamics analysis were carried out to discuss the prop shaft fixing method for the quad-copter mode. Based on the analysis results, the product design was carried out and the program was designed.

However, our design also carries many shortcomings. The design needs to be further optimized in terms of weight balance, material selection, power module selection, and body design. With limited budget and time for the development, we could largely depend on rule of thumb approaches. The best solution is, however, yet to be found and if that happens, our design and the VTOL efficiency would be further enhanced. Another limitation is that because the fuselage is made small, the aircraft can only carry a very small amount of payload (about $1 \mathrm{~kg}$ ). We need to make the size bigger, so it can carry a useful amount of onboard equipment and any commercial grade payload. In the future, we will use the CFD program instead of the above-mentioned kinetic analysis to test flights as fixed-wing mode and conduct further studies on path optimization. Stable VTOL UAV will be able to be used as reconnaissance, surveillance, and police drones for searching for missing persons and measuring traffic volumes. In this case, the combination of "airframe", which guarantees flight stability and VTOL convenience, as well as excellent video image transmit system would be important.

\section{Acknowledgements}

This work was supported by the Ajou University research fund.

\section{References}

[1] Jaemoo, K. (2013) Recent World Trend in VTOL Aircraft Development: Good Timing for Korean Aircraft Industry to Escape from Red Ocean. The Journal of Aerospace Industry, 79, 10-24.

[2] Panther-FE Fixed Wing VTOL Drone Demonstrated in Flight. https://doi.org/10.1080/028418501127346846

[3] Yu, S., Heo, J., Jeong, S. and Kwon, Y. (2016) Technical Analysis of VTOL UAV. Journal of Computer and Communications, 4, 92-97.

[4] The 16-Rotor Convertible Aircraft for Long-Range, High-Speed, Electric VTOL Commuting. 
http://newatlas.com/joby-s2-tilt-rotor-vtol-multirotor-aircraft-concept/40662/

[5] Kang, Y. (2015) Tilt-Rotor UAV System to Monitor Illegal Fishing. KIRI.

[6] High Eye VTOL Manufacture. http://www.higheye.nl/hef-32/

[7] Airbus to fly VSR700 VTOL UAV before End of the Year. http://www.janes.com/article/67281/airbus-to-fly-vsr700-vtol-uav-before-end-of-th e-year

[8] APID-55 VTOL Minature UAV. http://www.airforce-technology.com/projects/cybaero-apid55-uav/

[9] Watch Sony's First Aerosense Drone Take Flight. https://techcrunch.com/2015/08/24/watch-sonys-first-aerosense-drone-take-flight/

[10] JUMP 20 VTOL Unmanned Aerial Vehicle. http://www.naval-technology.com/projects/jump-20-vtol-unmanned-aerial-vehicle/

[11] Fisher, R.D. (2016) China Reveals Data on VD200 Flying-Wing VTUAV. IHS Jane's Defence Weekly, Washington DC, 30 March.

[12] Kim, D., Lee, B., Lee, Y. and Sung, S. (2016) VTOL UAV2 Design with Attitude Transition for High Maneuverability. ICROS Conference, 17-18.

[13] Yoshitani, N., Hashimoto, S., Kimura, T., Motohashi, K. and Ueno, S. (2009) Flight Control Simulators for Unmanned Fixed-Wing and VTOL Aircraft. ICROS Conference, 3211-3216.

[14] IT Choseun VTOL Drone (2016). http://it.chosun.com/news/article.html?no=2824299

[15] Jung, W., Lee, G. and Kim, J. (2003) Performance Estimation and Trade Study of VTOL Smart UAV Configurations in Pre-Concept Design Phase. KSAS Conference, 450-455.

[16] Naver VTOL Drone (2016). http://www.econovill.com/news/articleView.html?idxno=279193

Submit or recommend next manuscript to SCIRP and we will provide best service for you:

Accepting pre-submission inquiries through Email, Facebook, LinkedIn, Twitter, etc. A wide selection of journals (inclusive of 9 subjects, more than 200 journals)

Providing 24-hour high-quality service

User-friendly online submission system

Fair and swift peer-review system

Efficient typesetting and proofreading procedure

Display of the result of downloads and visits, as well as the number of cited articles

Maximum dissemination of your research work

Submit your manuscript at: http://papersubmission.scirp.org/

Or contact jcc@scirp.org 